\title{
Early Warning Factors of Death in COVID-19 Patients
}

\author{
Min SHANG ${ }^{1}$, Jie WEI ${ }^{2,3}$, Han-dong ZOU ${ }^{2,3}$, Qing-shan ZHOU ${ }^{2,3}$, Yun-ting ZHANG ${ }^{1}$, Chang-yong WANG ${ }^{2,3 \#}$ \\ ${ }^{I}$ Department of Respiratory and Critical Care Medicine, Renmin Hospital of Wuhan University, Wuhan 430060, China \\ ${ }^{2}$ Department of Intensive Care Medicine, Renmin Hospital of Wuhan University, Wuhan 430060, China \\ ${ }^{3}$ Department of Critical Care Medical Centre, Renmin Hospital of Wuhan University, Wuhan 430060, China
}

(C) Huazhong University of Science and Technology 2021

\begin{abstract}
Summary: The infectious coronavirus disease 2019 (COVID-19) has spread all over the world and been persistently evolving so far. The number of deaths in the whole world has been rising rapidly. However, the early warning factors for mortality have not been well ascertained. In this retrospective, single-centre cohort study, we included some adult inpatients ( $\geq 18$ years old) with laboratory-confirmed COVID-19 from Renmin Hospital of Wuhan University who had been discharged or had died by Apr. 8, 2020. Demographic, clinical and laboratory data at admission were extracted from electronic medical records and compared between survivors and non-survivors. We used univariable analysis, Cox proportional hazard model analysis and receiver operating characteristic (ROC) curve to explore the early warning factors associated with in-hospital death. A total of 159 patients were included in this study, of whom 86 were discharged and 73 died in hospital. Hypertension $(52.1 \%$ vs. $29.1 \%, P=0.003)$ and coronary heart disease $(28.8 \%$ vs. $12.8 \%$, $P=0.012$ ) were more frequent among non-survived patients than among survived patients. The proportions of patients with dyspnoea $(67.1 \%$ vs. $25.6 \%, P<0.001)$, chest distress $(58.9 \%$ vs. $26.7 \%$, $P<0.001)$ and fatigue $(64.4 \%$ vs. $25.6 \%, P<0.001)$ were significantly higher in the non-survived group than in the survived group. Regression analysis with the Cox proportional hazards mode revealed that increasing odds of in-hospital death were associated with higher IL-6 (odds ratio $10.87,95 \%$ CI $1.41-83.59 ; P=0.022)$, lactate $(3.59,1.71-7.54 ; P=0.001)$, older age $(1.86,1.03-3.38$; $P=0.041)$ and lower lymphopenia $(5.44,2.71-10.93 ; P<0.001)$ at admission. The areas under the ROC curve (AUCs) of IL-6, lymphocyte, age and lactate were $0.933,0.928,0.786$ and 0.753 respectively. The AUC of IL- 6 was significantly higher than that of age $(z=3.332, P=0.0009)$ and lactate $(z=4.441, P<0.0001)$ for outcome prediction. There was no significant difference between the AUCs of IL- 6 and lymphocyte for outcome prediction $(z=0.372, P=0.7101)$. It was concluded that the potential risk factors of higher IL-6, lactate, older age and lower lymphopenia at admission could help clinicians to identify patients with poor prognosis at an early stage.
\end{abstract}

Key words: coronavirus disease 2019; fatal cases; prognosis; mortality; early warning

Clusters of acute respiratory illness patients with unclear causes were found in some hospitals in Wuhan China in December 2019[1]. By January 7, 2020, Chinese scientists had isolated a novel coronavirus (severe acute respiratory syndrome coronavirus 2, SARS-CoV-2) from these patients with virus-infected pneumonia $^{[2,3]}$, which was later designated coronavirus disease 2019 (COVID-19) by WHO in February, $2020^{[4]}$. The disease has spread all over the world and been persistently evolving so $\mathrm{far}^{[5]}$. By 10:00 CET on April 1, 2020, a total of 740995 confirmed cases and 37277 deaths were reported from 204 foreign countries and regions on 6 continents plus the Diamond Princess international cruise ship. Among them, 161 countries and regions had local transmission ${ }^{[6]}$.

Although the number of new cases has

Min SHANG, E-mail: 57474557@qq.com

\#Corresponding author, E-mail: wcywhu@sina.com. significantly reduced in China because of intensive control procedures, the number in the rest of world is continuing to increase. It has been reported that the mortality of COVID-19 varies from $1.4 \%{ }^{[7]}$ to $4.3 \%{ }^{[8]}$ in different regions or hospitals. Although few deaths were found in mild COVID-19 patients, the mortality was obviously elevated among critical ill patients. Delays in identification of critical illness have been found to be independently associated with higher mortality ${ }^{[9]}$. There is an urgent need for the investigation for early risk factors of death in patients with COVID-19.

In this study, we aimed to compare the demographic, clinical, and laboratory features of patients at admission with different clinical outcomes to explore the investigation for early risk factors of death in patients with COVID-19. Through this study, we hope that the experience from China will assist the rest of the world in better risk stratification to achieve better outcomes for COVID-19 patients. 


\section{MATERIALS AND METHODS}

\subsection{Study Design and Participants}

This single-centre, retrospective, observational study was performed at the East Branch of Renmin Hospital of Wuhan University, a designated hospital to treat patients with SARS-CoV-2 pneumonia. We retrospectively analyzed 195 patients from Feb. 26, 2020 to Mar. 10, 2020, who had been diagnosed with COVID-19, according to the Guidance for Corona Virus Disease 2019 (6th edition) released by the National Health Commission of China ${ }^{[10]}$. Patients' living state was recorded while leaving hospital. Only patients who were discharged from hospital or had died were included in this study. Patients who died soon after admission and did not receive any laboratory testing were excluded. Patients younger than 18 years of age or with incomplete data were also excluded. This study was approved by the Ethics Commission of Renmin Hospital of Wuhan University. Written informed consent was waived due to the rapid emergence of this infectious disease.

\subsection{Data Collection}

Demographic information, preexisting comorbidities, signs and symptoms, laboratory test results, as well as outcome data, were obtained from electronic medical records. We collected data on demographics, medical history, underlying chronic diseases, symptoms and signs, laboratory findings during the hospital admission. The date of disease onset and admission to hospital, as well as dead or discharged date was recorded. The onset time was defined as the date when patients' any symptoms and signs first appeared. The clinical data were monitored up to 8 Apr. 2020.

\subsection{Laboratory Testing}

Patients' pharyngeal swab specimens were collected for extracting SARS-CoV-2 RNA. Viral nucleic acid was detected by real-time reverse-transcriptase polymerase chain reaction (RT-PCR) assay with a COVID-19 nucleic acid detection kit (BioGerm, China). All medical laboratory tests, including white blood cells (WBC), lymphocytes, C-reactive protein (CRP), procalcitonin (PCT), alanine aminotransferase (ALT), aspartate aminotransferase (AST), blood creatinine, blood urea nitrogen (BUN), cardiac troponin I (cTnI), D-dimer, albumin, lactate dehydrogenase $(\mathrm{LDH})$, lactate, interleukin 6 (IL-6), arterial partial pressure of oxygen $\left(\mathrm{PaO}_{2}\right)$, oxygenation index (OI) at admission were examined. The disease severity status at admission was also recorded. All medical laboratory tests were analyzed by the Clinical Laboratory of Renmin Hospital of Wuhan University.

\subsection{Statistical Analysis}

Continuous variables were expressed as mean \pm standard deviation (SD), and categorical variables were expressed as number (\%). We assessed differences between survivors and non-survivors using $t$ test or Mann-Whitney test depending on normal or abnormal data for continuous variables and $\chi^{2}$ test or Fishers' exact tests for categorical variables. Moreover, variables that showed significance $(P<0.05)$ were examined using Cox proportional hazard model analysis. Subsequently, the accuracy of each independent predictor was determined by each area under the receiver operating characteristic (ROC) curve (AUC). The Hosmer and Lemeshow test for goodness-of-fit statistics was used to check model adequacy. The AUCs of independent predictors were compared by Hanley-McNeil test. A value of $P<0.05$ was considered to be statistically significant. The SPSS Statistics 19.0 software, MedCalc 19.2 was applied for all analyses.

\section{RESULTS}

\subsection{Demographics Data and Clinical Characteristics}

Seventy-three non-survived and 86 survived cases of COVID-19 patients admitted to Renmin Hospital of Wuhan University were enrolled in this study. As shown in table 1, the median age of non-survived patients was 75 (interquartile range 68-82) years, which was significantly older than survived patients [61 (49-69) years). Overall, 53 (72.6\%) patients who died and 45 $(52.3 \%)$ who survived had at least one chronic medical condition. Hypertension $(52.1 \%$ vs. $29.1 \%, P=0.003)$ and coronary heart disease $(28.8 \% v s .12 .8 \%, P=0.012)$ were more frequent among non-survived patients than among survived patients.

Fever was one of the most prevalent symptoms at disease onset in both non-survived patients [63 (86.3\%)] and survived patients [73 (84.9\%)], and the proportions of patients reporting the symptoms in two groups were comparable. Other prevalent symptoms at onset of illness in non-survived patients included dyspnoea, cough, chest distress and fatigue. The proportions of patients with dyspnoea, chest distress and fatigue were significantly higher in the non-survived group than in the survived group. Also, there was significant difference in the disease severity status between the non-survived group and the survived group.

Among the non-survived patients, the median time from onset of symptoms to hospital admission was 11 (interquartile range 8-15) days, which was not significantly different from survived patients [12 (7$15)$ days]. The median time from onset of symptoms to death in non-survived patients was 18 (15-23) days, which was evidently shorter than the median time onset of symptoms to hospital discharge in survived patients [30 (24-35) days].

\subsection{Laboratory Findings}

As shown in table 2, patients in the non-survived group exhibited significantly higher WBC count, but lower lymphocyte count at admission than the survived 
Table 1 Baseline characteristics of patients with COVID-19 who survived or non-survived

\begin{tabular}{|c|c|c|c|c|}
\hline \multirow{2}{*}{ Baseline characteristics } & \multicolumn{3}{|c|}{$n(\%)$} & \multirow{2}{*}{$P$ value } \\
\hline & Total $(n=159)$ & Non-survivor $(n=73)$ & Survivor $(n=86)$ & \\
\hline \multicolumn{5}{|l|}{ Demographics and clinical characteristics } \\
\hline Median (IQR) age, years & $68(52-76)$ & $75(68-82)$ & $61(49-69)$ & $<0.001^{*}$ \\
\hline \multicolumn{5}{|l|}{ Sex } \\
\hline Female & $68(42.8)$ & $26(35.6)$ & $42(48.8)$ & 0.093 \\
\hline Male & $91(57.2)$ & $47(64.4)$ & $44(51.2)$ & \\
\hline Comorbidity & $98(61.6)$ & $53(72.6)$ & $45(52.3)$ & $0.009^{*}$ \\
\hline COPD & $11(6.9)$ & $8(11.0)$ & $3(3.5)$ & 0.064 \\
\hline Hypertension & $63(39.6)$ & $38(52.1)$ & $25(29.1)$ & $0.003^{*}$ \\
\hline Diabetes & $26(16.4)$ & $16(21.9)$ & $10(11.6)$ & 0.080 \\
\hline Coronary heart disease & $32(20.1)$ & $21(28.8)$ & $11(12.8)$ & $0.012^{*}$ \\
\hline Chronic liver disease & $6(3.8)$ & $3(4.1)$ & $3(3.6)$ & 1.000 \\
\hline Chronic kidney disease & $6(3.8)$ & $3(4.1)$ & $3(3.5)$ & 1.000 \\
\hline Malignancy & $10(6.3)$ & $3(4.1)$ & $7(8.0)$ & 0.474 \\
\hline \multicolumn{5}{|l|}{ Signs and symptoms } \\
\hline Fever (temperature $\geq 37.3^{\circ} \mathrm{C}$ ) & $136(85.5)$ & $63(86.3)$ & $73(84.9)$ & 0.800 \\
\hline Cough & $105(66.0)$ & $47(64.4)$ & $58(67.4)$ & 0.429 \\
\hline Chest distress & $66(41.5)$ & $43(58.9)$ & $23(26.7)$ & $<.0001^{*}$ \\
\hline Dyspnea & $71(44.7)$ & $49(67.1)$ & $22(25.6)$ & $<0.001^{*}$ \\
\hline Fatigue & $69(43.4)$ & $47(64.4)$ & $22(25.6)$ & $<0.001^{*}$ \\
\hline Diarrhea & $18(11.3)$ & $6(8.2)$ & $12(14.0)$ & 0.255 \\
\hline Abdominal pain & $3(1.9)$ & $3(4.1)$ & $0(0)$ & 0.189 \\
\hline Nausea vomiting & $8(5.0)$ & $3(4.1)$ & $5(5.8)$ & 0.900 \\
\hline Pharynx ministry unwell & $4(2.4)$ & $1(1.3)$ & $3(3.5)$ & 0.732 \\
\hline Chest pain & $2(1.3)$ & $0(0)$ & $2(2.3)$ & 0.500 \\
\hline Headache & $1(0.6)$ & $0(0)$ & $1(1.2)$ & 1.000 \\
\hline Myalgia & $11(6.9)$ & $2(2.7)$ & $9(10.5)$ & 0.056 \\
\hline Chills & $6(3.8)$ & $1(1.3)$ & $5(5.8)$ & 0.295 \\
\hline Median (IQR) respiratory rate (beats/min) & $20(19-25)$ & $21(18-27)$ & $20(19-23)$ & 0.645 \\
\hline $\begin{array}{l}\text { Median }(\mathrm{IQR}) \text { the highest } \\
\text { temperature }\left({ }^{\circ} \mathrm{C}\right)\end{array}$ & $38.2(37.6-38.8)$ & $38.5(38.0-38.9)$ & $38.0(37.5-38.7)$ & 0.132 \\
\hline Disease severity status & & & & $<0.001^{*}$ \\
\hline General & $37(23)$ & $3(4)$ & $34(39)$ & \\
\hline Severe & $47(30)$ & $9(12)$ & $38(44)$ & \\
\hline Critical & $75(47)$ & $61(84)$ & $14(16)$ & \\
\hline $\begin{array}{l}\text { Median (IQR) day from onset of } \\
\text { symptom to hospital admission (days) }\end{array}$ & $11(7-15)$ & $11(8-15)$ & $12(7-15)$ & 0.850 \\
\hline $\begin{array}{l}\text { Median(IQR) days from onset of } \\
\text { symptom to hospital discharge }\end{array}$ & $24(17-32)$ & $18(15-23)$ & $30(24-35)$ & $<0.001^{*}$ \\
\hline
\end{tabular}

COPD: chronic obstructive pulmonary disease; IQR: interquartile range. ${ }^{*} P<0.05$ was considered statistically significant.

group. At the time of admission, the CRP level was significantly higher in non-survived group than in the survived group. A significant difference in the PCT level was also found between the non-survived and survived groups. Concentrations of AST, BUN, blood creatinine, LDH, and D-dimer were markedly higher in non-survived patients than in survived patients. Albumin concentrations were significantly lower in non-survived patients than in survived patients. Lactate, cTnI and IL-6 concentrations were markedly higher in non-survived patients than in survived patients. $\mathrm{PaO}_{2}$ and OI were significantly lower in nonsurvived patients than in survived patients.

\subsection{Early Risk Factors of Death}

In univariable analysis, odds of in-hospital death was higher in patients with hypertension or coronary heart disease (table 3). The odds of in-hospital death was also higher in patients with dyspnoea, fatigue or chest distress. Old age, decreased lymphopenia, albumin, $\mathrm{PaO}_{2}$, OI, elevated leucocytosis, C-reactive protein, BUN, AST, LDH, D-dimer, lactate, blood creatinine, procalcitonin, cTnI and IL-6 were also associated with death (table 3 ).

We analyzed the complete data by the Cox proportional hazards regression analysis. It was found that older age, higher IL-6, lactate and lower lymphopenia at admission were associated with increased odds of death (table 3).

To determine and compare the accuracy of these factors in outcome prediction, ROC curve analysis 
Table 2 Laboratory findings of patients with COVID-19 who survived and non-survived at admission

\begin{tabular}{|c|c|c|c|c|c|}
\hline \multirow{2}{*}{ Findings } & \multirow{2}{*}{$\begin{array}{l}\text { Normal } \\
\text { Range }\end{array}$} & \multicolumn{3}{|c|}{ Median (IQR) } & \multirow{2}{*}{$P$ value } \\
\hline & & Total $(n=159)$ & Non-survivor $(n=73)$ & Survivor $(n=86)$ & \\
\hline WBC count $\left(\times 10^{9} / \mathrm{L}\right)$ & $3.5-9.5$ & $5.69(4.31-8.73)$ & $8.20(5.49-13.13)$ & $5.05(3.88-6.49)$ & $<0.001^{*}$ \\
\hline Lymphocyte count $\left(\times 10^{9} / \mathrm{L}\right)$ & $1.1-3.2$ & $1.19(0.62-3.06)$ & $0.61(0.39-0.88)$ & $2.79(1.72-4.18)$ & $<0.001^{*}$ \\
\hline $\mathrm{CRP}(\mathrm{mg} / \mathrm{L})$ & $0-10$ & $48.5(12.4-95.3)$ & $71.4(47.0-168.3)$ & $19.65(6.80-63.55)$ & $<0.001^{*}$ \\
\hline Procalcitonin (ng/mL) & $<0.1$ & $0.09(0.04-0.30)$ & $0.22(0.10-0.59)$ & $0.05(0.04-0.09)$ & $<0.001^{*}$ \\
\hline D-dimer $(\mathrm{mg} / \mathrm{L})$ & $0-0.55$ & $1.46(0.56-6.27)$ & $4.64(1.55-19.15)$ & $0.73(0.38-1.59)$ & $<0.001^{*}$ \\
\hline $\operatorname{ALT}(\mathrm{U} / \mathrm{L})$ & $7-40$ & $25.0(17.0-37.0)$ & $24.0(17.0-38.0)$ & $26.5(16.0-36.0)$ & 0.971 \\
\hline AST (U/L) & $13-35$ & $28(20-42)$ & $35(24-51)$ & $23(19-33)$ & $<0.001^{*}$ \\
\hline Albumin $(\mathrm{g} / \mathrm{L})$ & $40-55$ & $35.4(32.8-38.9)$ & $33.2(30.8-37.0)$ & $37.5(34.6-40.1)$ & $<0.001^{*}$ \\
\hline $\mathrm{BUN}(\mathrm{mmol} / \mathrm{L})$ & $2.6-7.5$ & $5.81(3.90-10.02)$ & $8.60(6.00-13.90)$ & $4.41(3.40-6.18)$ & $<0.001^{*}$ \\
\hline Serum creatine $(\mu \mathrm{mol} / \mathrm{L})$ & $41-73$ & $68(52-84)$ & $72(53-100)$ & $64(51-74)$ & $0.011^{*}$ \\
\hline $\mathrm{LDH}(\mathrm{U} / \mathrm{L})$ & $120-250$ & $313(232-464)$ & 457 (327-676) & $259(208-318)$ & $<0.001^{*}$ \\
\hline Lactate $(\mathrm{mmol} / \mathrm{L})$ & $0.5-1.5$ & $2.10(1.40-2.90)$ & $2.40(2.10-3.20)$ & $1.60(1.30-2.25)$ & $<0.001^{*}$ \\
\hline $\mathrm{PaO}_{2}(\mathrm{mmHg})$ & $80-100$ & $70(56-88)$ & $61(50-75)$ & $79(63-95)$ & $<0.001^{*}$ \\
\hline $\mathrm{OI}(\mathrm{mmHg})$ & $400-500$ & $200(145-300)$ & $175(114-200)$ & $287(187-313)$ & $<0.001^{*}$ \\
\hline CTnI (ng/mL) & $0-0.04$ & $0.012(0.005-0.062)$ & $0.047(0.013-0.245)$ & $0.005(0.005-0.009)$ & $<0.001^{*}$ \\
\hline$\underline{\mathrm{IL}-6}(\mathrm{pg} / \mathrm{mL})$ & $<10$ & $19.06(9.44-35.88)$ & $36.10(25.40-82.81)$ & $10.24(5.25-16.26)$ & $<0.001^{*}$ \\
\hline
\end{tabular}

WBC: white blood cells; CRP: C-reactive protein; ALT: alanine transaminase; AST: aspartate aminotransferase; BUN: blood urea nitrogen; LDH: lactate dehydrogenase; OI: oxygenation index; $\mathrm{PaO}_{2}$ : arterial partial pressure of oxygen; CTnI: cardiac troponin I; IL6: interleukin-6. " $P$ values indicate differences between non-survivors and survivors. $P<0.05$ was considered statistically significant.

was performed and the difference in AUCs was tested. The AUCs of IL-6, lymphocyte, age and lactate were 0.933, 0.928, 0.786, 0.753 (table 4, fig. 1). Hosmer and Lemeshow test showed a good model adequacy $\left(\chi^{2}=2.410, P=0.934\right)$. The AUC of IL- 6 was significantly higher than that of age $(z=3.332, P=0.0009)$ and lactate $(z=4.441, P<0.0001)$ for outcome prediction. There was no significant difference between the AUC of IL-6 and lymphocyte for outcome prediction $(z=0.372$, $P=0.7101$ ). With a cut-off value of 22.39 , IL-6 exhibited sensitivity of $84.62 \%$, and specificity of $88.89 \%$. With a cut-off value of 1.38 , lymphocyte exhibited sensitivity of $83.72 \%$, and specificity of $95.89 \%$.

\section{DISCUSSION}

This retrospective cohort study identified early risk factors of death in adult patients with COVID-19.

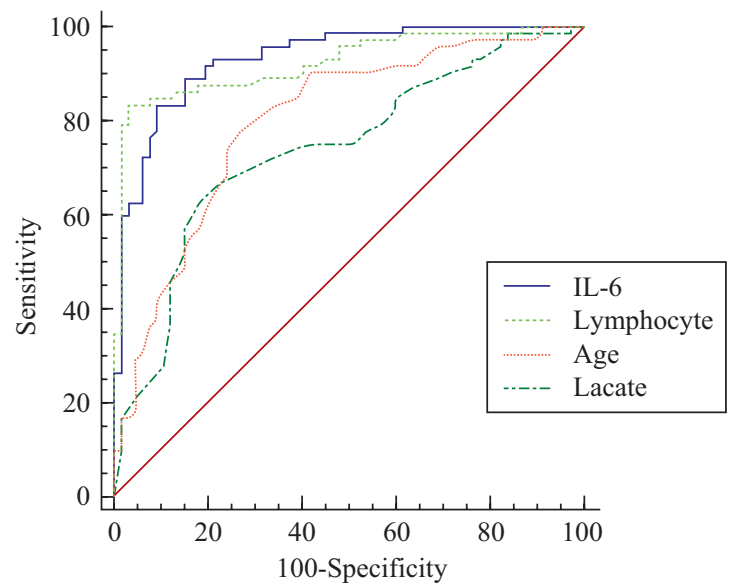

Fig. 1 ROC curves for predicting death in patients with COVID-19 IL-6: interleukin-6
In particular, older age, higher IL-6 and lactate, and lower lymphopenia at admission were associated with higher odds of in-hospital death. IL-6 which was not significantly different from lymphopenia performed better than other two parameters (age, lactate) in predicting adverse outcome in patients with COVID-19. Additionally, combidity of hypertension or coronary heart disease, dyspnoea chest distress or fatigue, decreased albumin, $\mathrm{PaO}_{2}$ and $\mathrm{OI}$, elevated levels of $\mathrm{WBC}, \mathrm{CRP}$, procalcitonin, BUN, LDH, D-dimer, blood creatinine, and cTnI at admission were more commonly seen in severe COVID-19 illness.

Advanced age is an independent predictor for an adverse outcome in patients developing SARS ${ }^{[11]}$. Zhou et al showed increased age was associated with higher odds of in-hospital death from SARS-Cov-2 infection ${ }^{[12]}$. Based on early statistical data of China, the case-fatality rate (CRF) in the patients over 60 years old was significantly higher than overall CRF, with 3.6\% CRF in patients aged 60-69 years, 8.0\% CRF in patients aged 70-79 years, $14.8 \%$ CRF in patients over 80 years ${ }^{[7]}$. Chen et al found that the risk of death rose with age ${ }^{[13]}$. Our research suggests that advanced age is an independent risk factor for death in patients with COVID-19, which is in accordance with the recent reports and provides additional evidence. It may be assumed that the respiratory mucosa in the aged patients is damaged and vulnerable to be invaded by the virus particles, which can infect other cells, trigger a series of immune responses and the production of cytokine storm in the body and lead to multiple organ dysfunction or even death ${ }^{[14,15]}$.

Lymphopenia was observed in $82.1 \%$ of COVID-19 patients $^{[7]}$. Previous findings indicated 
Table 3 Early risk factors of death in patients with COVID-19

\begin{tabular}{|c|c|c|c|c|}
\hline Factors & Univariable OR $(95 \% \mathrm{CI})$ & $P$ value & Multivariable OR $(95 \% \mathrm{CI})$ & $P$ value \\
\hline \multicolumn{5}{|c|}{ Demographics and clinical characteristics } \\
\hline \multicolumn{5}{|c|}{ Median (IQR) age (years) } \\
\hline \multicolumn{5}{|l|}{$\leq 65$} \\
\hline$>65$ & $5.84(2.91-11.76)$ & $<0.001^{*}$ & $1.86(1.03-3.38)$ & $0.041^{*}$ \\
\hline \multicolumn{5}{|l|}{ Comorbidity } \\
\hline Hypertension & $2.65(1.38-5.10)$ & $0.003^{*}$ & & \\
\hline Coronary heart disease & $2.75(1.22-6.19)$ & $0.014^{*}$ & & \\
\hline \multicolumn{5}{|l|}{ Signs and symptoms } \\
\hline Chest distress & $3.93(2.01-7.65)$ & $<0.001^{*}$ & & \\
\hline Dyspnea & $5.94(2.99-11.82)$ & $<0.001^{*}$ & & \\
\hline Fatigue & $5.24(2.66-10.39)$ & $<0.001^{*}$ & & \\
\hline \multicolumn{5}{|l|}{ Laboratory findings } \\
\hline \multicolumn{5}{|l|}{ WBC $\left(\times 10^{9} / \mathrm{L}\right)$} \\
\hline $4-10$ & 1 (ref) & & & \\
\hline$>10$ & $17.22(4.96-59.77)$ & $<0.001^{*}$ & & \\
\hline \multicolumn{5}{|l|}{ Lymphocyte count $\left(\times 10^{9} / \mathrm{L}\right)$} \\
\hline $1.1-3.2$ & 1 (ref) & & & \\
\hline$<1$ & $31.47(13.16-75.25)$ & $<0.001^{*}$ & $5.44(2.71-10.93)$ & $<0.001^{*}$ \\
\hline \multicolumn{5}{|l|}{$\mathrm{CRP}(\mathrm{mg} / \mathrm{L})$} \\
\hline $0-10$ & 1 (ref) & & & \\
\hline 10 & $9.03(2.99-27.23)$ & $<0.001^{*}$ & & \\
\hline \multicolumn{5}{|l|}{ Procalcitonin (ng/mL) } \\
\hline$\leq 0.1$ & 1 (ref) & & & \\
\hline$>0.1$ & $12.00(5.65-25.48)$ & $<0.001^{*}$ & & \\
\hline \multicolumn{5}{|l|}{ D-dimer (mg/L) } \\
\hline $0-0.55$ & 1 (ref) & & & \\
\hline$>0.55$ & $7.37(2.68-20.29)$ & $<0.001^{*}$ & & \\
\hline \multicolumn{5}{|l|}{$\operatorname{AST}(\mathrm{U} / \mathrm{L})$} \\
\hline$\leq 40$ & 1 (ref) & & & \\
\hline$>40$ & $5.09(2.37-10.93)$ & $<0.001^{*}$ & & \\
\hline \multicolumn{5}{|l|}{ Albumin (g/L) } \\
\hline$\geq 40$ & 1 (ref) & & & \\
\hline$<40$ & $2.79(1.16-6.73)$ & $0.022^{*}$ & & \\
\hline \multicolumn{5}{|l|}{ BUN (mmol/L) } \\
\hline $2.6-7.5$ & 1 (ref) & & & \\
\hline$>7.5$ & $8.52(4.01-18.10)$ & $<0.001^{*}$ & & \\
\hline \multicolumn{5}{|l|}{ Serum creatine $(\mu \mathrm{mol} / \mathrm{L})$} \\
\hline $41-73$ & 1 (ref) & & & \\
\hline$>73$ & $2.39(1.23-4.63)$ & $0.010^{*}$ & & \\
\hline \multicolumn{5}{|l|}{ LDH (U/L) } \\
\hline $120-250$ & $1(\mathrm{ref})$ & & & \\
\hline$>250$ & $7.07(3.03-16.49)$ & $<0.001^{*}$ & & \\
\hline \multicolumn{5}{|l|}{ Lactate $(\mathrm{mmol} / \mathrm{L})$} \\
\hline $0.5-1.5$ & 1 (ref) & & & \\
\hline$>1.5$ & $6.67(2.83-15.76)$ & $<0.001^{*}$ & $3.59(1.71-7.54)$ & $0.001^{*}$ \\
\hline \multicolumn{5}{|l|}{$\mathrm{PaO}_{2}(\mathrm{mmHg})$} \\
\hline $80-100$ & $1(\mathrm{ref})$ & & & \\
\hline$<80$ & $3.56(1.77-7.15)$ & $<0.001^{*}$ & & \\
\hline OI (mmHg) & & & & \\
\hline$\geq 300$ & 1 (ref) & & & \\
\hline$<300$ & $15.00(5.03-44.76)$ & $<0.001^{*}$ & & \\
\hline CTnI (ng/mL) & & & & \\
\hline $0-0.04$ & 1 (ref) & & & \\
\hline$>0.04$ & $25.59(7.34-89.24)$ & $<0.001^{*}$ & & \\
\hline IL-6 (pg/mL) & & & & \\
\hline$<10$ & 1 (ref) & & & \\
\hline$\geq 10$ & $67.45(8.92-510.01)$ & $<0.001^{*}$ & $10.87(1.41-83.59)$ & $0.022^{*}$ \\
\hline
\end{tabular}

IQR: interquartile range; WBC: white blood cells; CRP: C-reactive protein; AST: aspartate aminotransferase; BUN: blood ureanitrogen; LDH: lactate dehydrogenase; $\mathrm{PaO}_{2}$ : arterial partial pressure of oxygen; OI: oxygenation index; CTnI: cardiac troponin I; IL-6: interleukin-6. ${ }^{*} P$ values indicate differences between non-survivor and survivor. $P<0.05$ was considered statistically significant. 
Table 4 Predictive value of early risk factors of death in patients with COVID-19

\begin{tabular}{lccccccc}
\hline Indicators & AUC & Standard error & $95 \%$ CI & $P$ value & Optimal critical value & Sensitivity (\%) & Specificity (\%) \\
\hline Age (years) & 0.786 & 0.037 & $0.714-0.847$ & $<0.001^{*}$ & 69 & 81.40 & 65.75 \\
IL-6 $(\mathrm{pg} / \mathrm{mL})$ & 0.933 & 0.019 & $0.881-0.968$ & $<0.001^{*}$ & 22.39 & 84.62 & 88.89 \\
Lymphocyte count $\left(\times 10^{9} / \mathrm{L}\right)$ & 0.928 & 0.021 & $0.877-0.963$ & $<0.001^{*}$ & 1.38 & 83.72 & 95.89 \\
Lactate $(\mathrm{mmol} / \mathrm{L})$ & 0.753 & 0.040 & $0.675-0.820$ & $<0.001^{*}$ & 2.00 & 68.75 & 77.61 \\
\hline
\end{tabular}

IL-6: interleukin-6. $P$ values indicate differences between non-survivor and survivor. ${ }^{*} P<0.05$ was considered statistically significant.

that lymphocytopenia is seen more often in nonsurvivors with COVID-19 ${ }^{[8,16]}$. Yang et al found that lymphocytopenia occurred in more than $80 \%$ of critically ill patients ${ }^{[17]}$. Chen et al found that deceased patients had more severe and persistent lymphopenia than the recovered patients ${ }^{[13]}$. Deng et al found that the lymphocyte count in the death group was less than the recovered group and decreased during hospitalization ${ }^{[18]}$. Our research demonstrates that lower lymphopenia at admission not only is an independent risk factor for death in patients with COVID-19, but also shows good predictive power for in-hospital death, which is consistent with the previous studies. Wang et al found that lymphocyte count decreased evidently in the dead group and increased lymphocyte count was predictive of better outcomes, which confirms our conclusion $^{[19]}$. Zhou et al found that total lymphocyte count was a risk factor associated with disease progression in patients with COVID-19 by using binary logistic regression model ${ }^{[20]}$. Lymphopenia (peripheral blood lymphocyte count $<0.8 \times 10^{9} / \mathrm{L}$ ) was reported to be one of simple and reliable mortality predictors for influenza pneumonia ${ }^{[21]}$. It has been speculated that lymphocytes may be killed by coronavirus due to the damage of the cytoplasmic components or apoptosis ${ }^{[22,}$ ${ }^{23}$. Chen et al found that SARS-CoV-2 infection induced lymphopenia, particularly in $\mathrm{CD} 4^{+}$and $\mathrm{CD} 8^{+} \mathrm{T}$ cells, as well as suppressed IFN- $\gamma$ production by $\mathrm{CD}^{+}{ }^{+} \mathrm{T}$ cells, which might be connected with disease severity of COVID-19 ${ }^{[24]}$. To investigate the relationship between lymphocytes, especially $\mathrm{T}$ lymphocytes and the disease severity in patients with SARS-CoV-2 infection thoroughly, is of great significance for the clinical treatment of COVID-19 patients.

Li et al found that the levels of the lastly tested lactate $(100 \%)$ of dead patients with COVID-19 were increased as compared with those at the first test, which suggests that the rising of lactate levels can be used as an indicator of disease progression ${ }^{[25]}$. Our research suggests that higher lactate is an independent risk factor for death in patients with COVID-19. There were few relevant studies on the role of lactate in prognostic assessment in patients with COVID-19, which might be related to lack of lactate test at admission. Studies have shown that routine measurement of lactate in patients with infection can impact clinical assessment of mortality risk ${ }^{[26]}$. Hsu et al found that high lactate levels were independent predictors of death in critically ill influenza patients ${ }^{[27]}$. We performed lactate test for patients at admission, which can identify critically ill patients earlier and create conditions for reducing mortality.

A major pathophysiological mechanism implicated in severe COVID-19 is an aberrant host immune response resulting in an excessive cytokine and chemokine release known as "cytokine storm" $[28,29]$. IL-6 is one of the key factors, which contributes to the cytokinemediated damage and inflammation ${ }^{[29]}$. Plasma levels of IL-6 have been found to be elevated in patients with COVID-19, which correlates with disease severity and multiorgan failure ${ }^{[30,31]}$. Our research demonstrates that higher IL-6 is an independent risk factor for death in patients with COVID-19, which also has considerable predictive value for outcome prediction. Many studies have shown that IL- 6 can be used as an independent factor to predict the severity of COVID-19, which is consistent with our findings ${ }^{[32-34]}$. Hu et al reported that cytokine storm with rapidly elevated IL-6 indicates sudden death in patients with critical COVID-19, which further indicates the importance of dynamic monitoring of IL-6 in early identification of potential death in patients with COVID-19 ${ }^{[35]}$.

To our surprise, lymphocyte was found to have high predictive value for predicting adverse outcome in patients with COVID-19, which was not significantly different from IL-6 and performed better than other two parameters (age, lactate). Lymphocyte counts are not only easy to measure, inexpensive, but also highly valuable for the prediction of COVID-19 death, which is of great significance in the evaluation of condition and therapeutic effect.

Our study has some limitations. First, owing to the retrospective study design, not all laboratory tests were done in all patients, including $\mathrm{N}$ terminal pro $\mathrm{B}$ type natriuretic peptide (NT-proBNP). Therefore, their role might be underestimated in predicting in-hospital death. Second, patients were sometimes transferred late in their illness to our hospitals. Lack of timely effective therapy might have also contributed to the poor outcomes in some patients. Last, our sample was relatively small and not all of key prognostic factors were included in the study. Therefore, further study is warranted to gain a better understanding of early warning factors of death for patients with COVID-19, which ultimately may help doctors identify critically ill patients and treat them earlier to reduce mortality. 
In conclusion, the COVID-19 patients that are accompanied with older age, higher IL-6 and lactate, and lower lymphopenia at admission are at high risk of death. The patients who have combidity of hypertension or coronary heart disease, dyspnoea, chest distress, or fatigue, decreased albumin, $\mathrm{PaO}_{2}$ and OI, elevated levels of WBC, CRP, procalcitonin, BUN, LDH, D-dimer, blood creatinine, and cTnI at admission should not be neglected either.

\section{Conflict of Interest Statement}

The authors declare no potential conflict of interest relevant to this article.

\section{REFERENCES}

1 WHO. Novel coronavirus-China. 2020.Available at: https:// www.who.int/emergencies/diseases/novel-coronavirus-2019.

2 Phelan AL, Katz R, Gostin LO. The Novel Coronavirus Originating in Wuhan, China: Challenges for Global Health Governance. JAMA, 2020,323(8):709-710

3 Coronaviridae Study Group of the International Committee on Taxonomy of Viruses. The species Severe acute respiratory syndrome-related coronavirus: classifying 2019-nCoV and naming it SARS-CoV-2. Nat Microbiol, 2020,5(4):536-544

4 Chan JW, Ng CK, Chan YH, et al. Short term outcome and risk factors for adverse clinical outcomes in adults with severe acute respiratory syndrome (SARS). Thorax, 2003,58(8):686-689

5 Zhu N, Zhang D, Wang W, et al. A Novel Coronavirus from Patients with Pneumonia in China, 2019. N Engl J Med, 2020,382(8):727-733

6 China CDC Weekly. Novel coronavirus-China. 2020. Http://weekly.chinacdc.cn/index.htm.

7 Guan WJ, Ni ZY, Hu Y, et al. Clinical Characteristics of Coronavirus Disease 2019 in China. N Engl J Med, 2020,382(18):1708-1720

8 Wang D, Hu B, Hu C, et al. Clinical Characteristics of 138 Hospitalized Patients With 2019 Novel Coronavirus-Infected Pneumonia in Wuhan, China. JAMA, 2020,323(11):1061-1069

9 Boniatti MM, Azzolini N, Viana MV, et al. Delayed medical emergency team calls and associated outcomes. Crit Care Med, 2014,42(1):26-30

10 New coronavirus pneumonia prevention and control program (6th ed) (in Chinese). 2020. Available at: http:// www.nhc.gov.cn/xcs/zhengcwj/202002/8334a8326dd9 4d329df351d7da8aefc2/files/b218cfeb1bc54639af227f 922bf6b817.pdf

11 Lee N, Hui D, Wu A, et al. A major outbreak of severe acute respiratory syndrome in Hong Kong. N Engl J Med, 2003,348(20):1986-1994

12 Zhou F, Yu T, Du R, et al. Clinical course and risk factors for mortality of adult inpatients with COVID-19 in Wuhan, China: a retrospective cohort study. Lancet, 2020,395(10229):1054-1062

13 Chen T, Wu D, Chen H, et al. Clinical characteristics of 113 deceased patients with coronavirus disease 2019: retrospective study. BMJ, 2020,368:m1091

14 Cho SJ, Stout-Delgado HW. Aging and Lung Disease. Annu Rev Physiol, 2020,82:433-459
15 Guo YR, Cao QD, Hong ZS, et al. The origin, transmission and clinical therapies on coronavirus disease 2019 (COVID-19) outbreak --- an update on the status. Mil Med Res, 2020,7(1):11

$16 \mathrm{Du}$ Y, Tu L, Zhu P, et al. Clinical Features of 85 Fatal Cases of COVID-19 from Wuhan. A Retrospective Observational Study. Am J Respir Crit Care Med, 2020, 201(11):1372-1379

17 Yang X, Yu Y, Xu J, et al. Clinical course and outcomes of critically ill patients with SARS-CoV-2 pneumonia in Wuhan, China: a single-centered, retrospective, observational study. Lancet Respir Med, 2020,8(5):475481

18 Deng Y, Liu W, Liu K, et al. Clinical characteristics of fatal and recovered cases of coronavirus disease 2019 in Wuhan, China: a retrospective study. Chin Med J (Engl), 2020,133(11):1261-1267

19 Wang L, He W, Yu X, et al. Coronavirus disease 2019 in elderly patients: Characteristics and prognostic factors based on 4-week follow-up. J Infect, 2020,80(6):639645

20 Zhou Y, Zhang Z, Tian J, et al. Risk factors associated with disease progression in a cohort of patients infected with the 2019 novel coronavirus. Ann Palliat Med, 2020,9(2):428-436

21 Shi SJ, Li H, Liu M, et al. Mortality prediction to hospitalized patients with influenza pneumonia: $\mathrm{PO}_{2} /$ $\mathrm{FiO}_{2}$ combined lymphocyte count is the answer. Clin Respir J, 2017,11(3):352-360

$22 \mathrm{Gu} \mathrm{J}$, Gong E, Zhang B, et al. Multiple organ infection and the pathogenesis of SARS. J Exp Med, 2005,202(3):415-424

23 Chu H, Zhou J, Wong BH, et al. Middle East Respiratory Syndrome Coronavirus Efficiently Infects Human Primary $\mathrm{T}$ Lymphocytes and Activates the Extrinsic and Intrinsic Apoptosis Pathways. J Infect Dis, 2016,213(6):904-914

24 Chen $\mathrm{G}, \mathrm{Wu} \mathrm{D}$, Guo W, et al. Clinical and immunological features of severe and moderate coronavirus disease 2019. J Clin Invest, 2020,130(5):2620-2629

25 Li X, Wang L, Yan S, et al. Clinical characteristics of 25 death cases with COVID-19: A retrospective review of medical records in a single medical center, Wuhan, China. Int J Infect Dis, 2020,94:128-132

26 Trzeciak S, Dellinger RP, Chansky ME, et al. Serum lactate as a predictor of mortality in patients with infection. Intensive Care Med, 2007,33(6):970-977

27 Hsu JC, Lee IK, Huang WC, et al. Clinical Characteristics and Predictors of Mortality in Critically Ill Influenza Adult Patients. J Clin Med, 2020,9(4):1073

$28 \mathrm{Fu} \mathrm{B}, \mathrm{Xu} \mathrm{X}$, Wei H. Why tocilizumab could be an effective treatment for severe COVID-19?. J Trans1 Med, 2020,18(1):164

29 Chen X, Zhao B, Qu Y, et al. Detectable Serum Severe Acute Respiratory Syndrome Coronavirus 2 Viral Load (RNAemia) Is Closely Correlated With Drastically Elevated Interleukin 6 Level in Critically Ill Patients With Coronavirus Disease 2019. Clin Infect Dis, 2020, 71(8):1937-1942

30 Liu B, Li M, Zhou Z, et al. Can we use interleukin-6 (IL-6) blockade for coronavirus disease 2019 (COVID19)-induced cytokine release syndrome (CRS)?. J 
Autoimmun, 2020,111:102452

31 Ruan Q, Yang K, Wang W, et al. Clinical predictors of mortality due to COVID-19 based on an analysis of data of 150 patients from Wuhan, China. Intensive Care Med, 2020,46(5):846-848

32 Liu F, Li L, Xu M, et al. Prognostic value of interleukin-6, $\mathrm{C}$-reactive protein, and procalcitonin in patients with COVID-19. J Clin Virol, 2020,127:104370

33 Zhu Z, Cai T, Fan L, et al. Clinical value of immuneinflammatory parameters to assess the severity of coronavirus disease 2019. Int J Infect Dis, 2020,95:332339

34 Liu Z, Li J, Chen D, et al. Dynamic Interleukin-6 Level Changes as a Prognostic Indicator in Patients With COVID-19. Front Pharmacol, 2020,11:1093

$35 \mathrm{Hu} \mathrm{Z}$, Li S, Song X. Cytokine storm with rapidly elevated interleukin-6 indicates sudden death in patients with critical COVID-19. Cytokine Growth Factor Rev, 2020,S1359-6101(20)30200-8

(Received Apr. 25, 2020; accepted Oct. 28, 2020) 